\title{
Energy dissipation channels in reactive and non-reactive scattering at surfaces
}

\author{
M. Alducin, R. Díez Muiño, and J. I. Juaristi
}

\begin{abstract}
One of the main challenges in theoretical gas-surface studies is to incorporate into the dynamics energy exchange to both lattice vibrations and electronic excitations, keeping the accuracy of a multidimensional ab-initio potential energy surface for describing the gas/metal interaction. In this chapter, we review some recent advances in the subject and will present a theoretical framework recently developed that allows to evaluate within a full dimensional dynamics the combined contribution of both excitation mechanisms. This objective has been accomplished by combining the Generalized Langevin Oscillator model for phonon excitations and the Local Density Friction Approximation for electronic excitations. The inclusion of both effects allows one to address such fundamental questions as which is the relative importance of phonon and electron-hole pair excitations as energy dissipation channels and to what extent the adiabatic calculation can capture the basic physics of the dynamics and provide accurate results. Results on several systems and on different elementary gas-surface processes (dissociation, scattering, and molecular adsorption) are used to enrich the discussion. We show that, even when the energy dissipated is quantitatively significant, important aspects of the scattering dynamics are well captured by the adiabatic approximation.
\end{abstract}

\footnotetext{
M. Alducin

Centro de Física de Materiales CSIC-UPV/EHU, San Sebastián, Spain

Donostia International Physics Center DIPC, San Sebastián, Spain

e-mail: wapalocm@ehu.es,

R. Díez Muiño

Centro de Física de Materiales CSIC-UPV/EHU, San Sebastián, Spain

Donostia International Physics Center DIPC, San Sebastián, Spain

e-mail: rdm@ehu.es

J. I. Juaristi

Departamento de Física de Materiales UPV/EHU, San Sebastián, Spain

Centro de Física de Materiales CSIC-UPV/EHU, San Sebastián, Spain

Donostia International Physics Center DIPC, San Sebastián, Spain

e-mail: josebainaki.juaristi@ehu.es
} 


\section{Introduction}

An accurate theoretical description of the dynamical interaction between atoms and small molecules in the gas phase and clean surfaces usually requires first-principles calculations, as well as the inclusion of the full dimensionality of the problem. Although less refined approximations have been very useful in the past and still help in the understanding of surface chemistry phenomena, the jump from qualitative to quantitative analysis demands high precision in the numerical evaluation of intricate energy topographies.

An effective approach is the calculation of the potential energy surface (PES) of the system prior to launching the dynamics. The obtention of the gas-surface PES is generally based on strict approximations. PESs are computed in the adiabatic approximation in which inelastic processes are neglected. The interaction energy between slowly moving gas-phase species and surface atoms, for a given position, is considered as that of the ground state. Neither the energy exchange with phonons nor the possibility of electron-hole pair creation are included. Despite the approximations involved, adiabatic descriptions of reactive and non-reactive processes at surfaces have proven to be very successful in a wide variety of systems. In the particular case of metal surfaces, the reflection and dissociative adsorption of small molecules such as $\mathrm{H}_{2}, \mathrm{~N}_{2}$, and in some cases $\mathrm{O}_{2}$ have been shown to be well described in the adiabatic picture [1-7]. A more detailed account of the expected precision of the adiabatic approximation can be found in other chapters of this book.

The quest for improved accuracy requires further advance in the theoretical description of all processes involved in the dynamics, including those associated with energy exchange. In addition, there are systems for which energy exchange can be indeed relevant. Electron excitations and phonons are, in general, the most prevalent mechanisms. Concerning electronic excitations, there is ample experimental evidence showing that they do arise in gas-surface experiments [8-11]. For this reason they have been the focus of much theoretical research in recent years, and different models have been developed to include this energy dissipation channel [12-16]. A remaining challenge consisted in being able to model electronic excitations in gassurface dynamics without losing the accuracy achieved in full-dimensional adiabatic calculations. This has been recently accomplished with the so-called local density friction coefficient approximation (LDFA) [14]. The LDFA framework has finally allowed to incorporate electronic excitations in multidimensional dynamics using accurate ab-initio PESs.

Theoretical activity has been also broad [17-20] in the study of the energy exchange between incident atoms and molecules and the surface lattice. For instance, Manson and coworkers in a series of papers have studied extensively the energy exchange between impinging atoms and molecules with surfaces, using a semiclassical approximation [19]. In these works, model surfaces were considered such as a smooth flat vibrating surface, a collection of discrete scatterers, and even an intermediate case in which surface corrugation was modeled via a corrugation parameter [21]. This approach has shown to be very successful to understand energy exchange in case of nonreactive rare gas atom scattering with surfaces, for which the 
smooth surface constitutes a reasonable approximation [22,23]. Even for the case of the scattering of reactive molecules, the model has been applied and valuable information has been obtained $[24,25]$. Nevertheless, for reactive species for which the multidimensional PES is very corrugated and intricate, the accuracy of such simplified molecule-surface interaction potentials can be questionable. In these cases, a more realistic treatment of the interaction, at least at the level of DFT, is necessary. In this respect, the Generalized Langevin Oscillator model (GLO) $[17,18]$ is a valuable tool to incorporate energy exchange with the lattice phonons on top of a multidimensional ab-initio PES [26,27].

A theoretical model has been recently introduced that, keeping the accuracy of a multidimensional ab-initio DFT-PES for the gas-particle/metal interaction, incorporates in the dynamics energy exchange to both lattice vibrations and electronic excitations [28]. For the reasons explained above, such a model is necessary for a complete realistic description of the gas-surface interaction. This has been done by combining the LDFA for electronic excitations and the GLO for phonon excitations/deexcitations. This approach will be denoted as the GLO \& LDFA model. In this chapter, we will use some of the capabilities of this model to review and discuss the role of electronic friction and phonons in elementary reactive processes arising in some representative systems.

This chapter is organized as follows: Section 2 is devoted to the description of the LDFA, the GLO and the GLO \& LDFA models to incorporate electronic and phonon excitation effects in gas-surface dynamics, their physical basis and how they are implemented in multidimensional ab-initio dynamics, in Section 3 these methods will be applied to some representative systems to show what kind of information can be gained on the role of electronic and phonon excitations in some gas-surface processes and to explore up to what point the adiabatic approximation represents a good approximation for some of these problems, and, finally, Section 4 is devoted to summarize the main conclusions that can be obtained from these studies.

\section{Theoretical Framework}

This section describes the theoretical models developed to account for electronic excitations and energy exchange and dissipation effects in the coupling of the molecule to the lattice vibrations. All these models constitute an efficient framework to include such nonadiabatic processes in dynamics simulations run on an accurate multidimensional potential energy surface. The section starts by introducing the LDFA of Juaristi and co-workers [14] that incorporates the role of low energy electronhole pair excitations as a friction force experienced by the impinging gas-atom or -molecule. The section continues with the GLO model proposed by Adelman and Doll [17] and Tully [18] that accounts for lattice vibrations and their effect is gassurface dynamics. The sections ends up with the GLO\&LDFA model that incorporates in the equations of motion the electronic and phonon excitations by naturally combining the LDFA for the former and the GLO for the latter. 


\subsection{Local density approximation for electronic friction}

The LDFA allows to incorporate electronic excitations in the interaction of low energy atoms and molecules with metal surfaces. An important characteristic of the model is that it can be implemented in multidimensional dynamics calculations performed on top of ab-initio Potential Energy Surfaces (PES). This condition is necessary, since it has been widely proven that a realistic simulation of reactivity and/or scattering of gas species interacting with metal surfaces at least requires the inclusion of all degrees of freedom of the projectile in the dynamics and a realistic gas-metal interaction potential. Additionally, the model must take into account the fact that a low velocity atomic particle represents a strong perturbation for the electronic system. This means that a perturbative treatment within linear response theory of the projectile-surface interaction is not adequate for typical elementary gas-surface processes, and that a nonlinear screening model that treats the interaction to all orders in the atomic charge $\mathrm{Z}_{1}$ is required [29].

All these requirements are fulfilled by the Local Density Friction Approximation (LDFA) $[14,30]$. As a first step, one calculates the electronic density $n\left(\mathbf{r}_{i}\right)$ of the bare surface (i.e., without the projectile) at each position $\mathbf{r}_{i}$ of the trajectory followed by each of the atomic constitutes of the impinging projectile (the gas-atom or -molecule). The surface electronic density $n\left(\mathbf{r}_{i}\right)$ is calculated ab-initio using DFT. The energy lost by the projectile due to electron-hole pair excitations is, then, calculated in terms of a friction coefficient that, at each point of the trajectory, is approximated by that corresponding to an electron gas with electronic density $n_{0}=n\left(\mathbf{r}_{i}\right)$. In other words, each atomic particle interacting with the metal surface is considered, at each point of its trajectory to be embedded in a free electron gas with electronic density equal to that of the bare surface at this point. What remains is to calculate the electronic frictional force that an atomic particle suffers in such free electron gas system.

In principle, to calculate the perturbation created in an electron gas by a moving projectile and the subsequent energy loss, one may try to obtain the induced density as a function of time through the time-dependent Kohn-Sham scheme of the time dependent density functional theory (TDDFT). However, as it was demonstrated in Ref. [31], in the adiabatic limit, valid for the low projectile velocities under consideration, the exact time-dependent density can be obtained in a static Kohn-Sham formulation.

The demonstration is based on the concept of the shift of the Fermi sphere developed by Schönhammer $[32,33]$. In this approach, the time-dependent Schrödinger equation for the electrons interacting with the moving projectile, is transformed to a time-independent equation by means of a Galileo transformation to the projectile reference frame. This allows one to obtain a general expression for the energy dissipation rate in an interacting electron gas that reduces to a simple integral involving one-electron force matrix elements with scattering states for non-interacting electrons [32]. Though the obtained result is exact for arbitrary velocities and strength of the interacting potential, there exist practical problems to evaluate it because it requires the knowledge of the exact dynamic non-spherically symmetric projectile- 
electron potential and the component parallel to the velocity of the transport cross section (a vectorial quantity when the potential is not spherical). The only exact calculations within DFT performed for arbitrary velocities, using this scheme, have been presented in Ref. [31] for protons and antiprotons. However, taking the limit for vanishing projectile velocities, one obtains that the resulting effect of electronic excitations is a mere friction force proportional to the projectile velocity. In this case, the corresponding friction coefficient is proportional to the transport cross section evaluated at the Fermi level of the spherically symmetric static projectile-electron potential [33]. When this potential is the Kohn-Sham static potential, this approach constitutes the exact low velocity limit of the TDDFT for an atomic projectile traveling through jellium [31]. We stress that this is the limit of interest for thermal and hyperthermal projectiles due to their low velocity as compared with the Fermi velocity of a metal which is, typically, in the range of 0.3-1.3 Böhr velocities. In fact, the model has been successfully used to calculate the energy loss of ion projectiles interacting with the bulk and the surface of metals up to velocities of the order of the Fermi velocity [34-36].

Briefly, within this scheme the dissipative force on an atom traveling with velocity $\mathbf{v}$ through a free electron gas of density $n_{0}$ can be obtained as follows [34]:

$$
\mathbf{F}_{\text {diss }}=-\eta \mathbf{v}=n_{0} \mathbf{v} k_{F} \sigma_{t r}\left(k_{F}\right),
$$

where $\eta$ is the friction coefficient, $k_{F}$ the Fermi momentum and $\sigma_{t r}\left(k_{F}\right)$ the transport cross section at the Fermi level. The product $k_{F} \sigma_{t r}\left(k_{F}\right)$ is the integrated scattering rate for momentum transfer which governs the dissipative process. Therefore, one can interpret the dissipative force described by Eq. (1) as the result of the momentum transfer per unit time to a uniform current of independent electrons $\left(n_{0} v\right)$, scattered by a fixed impurity potential. Note that the friction coefficient reads $\eta=n_{0} k_{F} \sigma_{t r}\left(k_{F}\right)$. The transport cross-section is calculated to all orders in the nuclear charge $\mathrm{Z}_{1}$ of the atom in terms of the scattering phase-shifts at the Fermi level $\left[\delta_{l}\left(k_{F}\right)\right][34,35]$ :

$$
\sigma_{t r}\left(k_{F}\right)=\frac{4 \pi}{k_{F}^{2}} \sum_{l=0}^{\infty}(l+1) \sin ^{2}\left[\delta_{l}\left(k_{F}\right)-\delta_{l+1}\left(k_{F}\right)\right] .
$$

In these equations the scattering potential is the screening potential induced by the impurity in the electron-gas system. As explained above, this potential is obtained within the static density functional theory for an impurity with nuclear charge $\mathrm{Z}_{1}$ embedded in an homogenous electron gas of density $n_{0}$ [37]. In this way, the model includes non linear effects both in the medium response to the atomic potential (nonlinear screening) and in the calculation of the relevant cross-section for the energy loss process.

For implementation in a molecular dynamics simulation it is very convenient to use an analytical fitting expression that provides the friction coefficient as a function of the electronic density. An excellent fitting function to the DFT friction coefficients for $\mathrm{H}, \mathrm{N}$ and $\mathrm{O}$ atoms is provided by the following expression: 


$$
\eta\left(r_{s}\right)=\sum_{i=1}^{n} A_{i} r_{s}^{B_{i}} \exp \left(-C_{i} r_{s}\right)
$$

where $r_{s}$ is the free electron radius $\left(\frac{1}{n_{0}}=\frac{4}{3} \pi r_{s}^{3}\right)$, and $A_{i}, B_{i}$ and $C_{i}$ are fitting parameters. In this expression both $\eta$ and $r_{s}$ are given in atomic units $\left(e=m_{e}=\hbar=1\right)$. The values of the fitting parameters for $\mathrm{H}, \mathrm{N}$ and $\mathrm{O}$ atoms are listed in Table 1. Note that the fitting function consists of one term $(n=1)$ for the $\mathrm{H}$ atom and two terms $(n=2)$ for the $\mathrm{N}$ and $\mathrm{O}$ atoms. The quality of the analytical fitting expression of Eq. (3) is shown in Fig. 1.
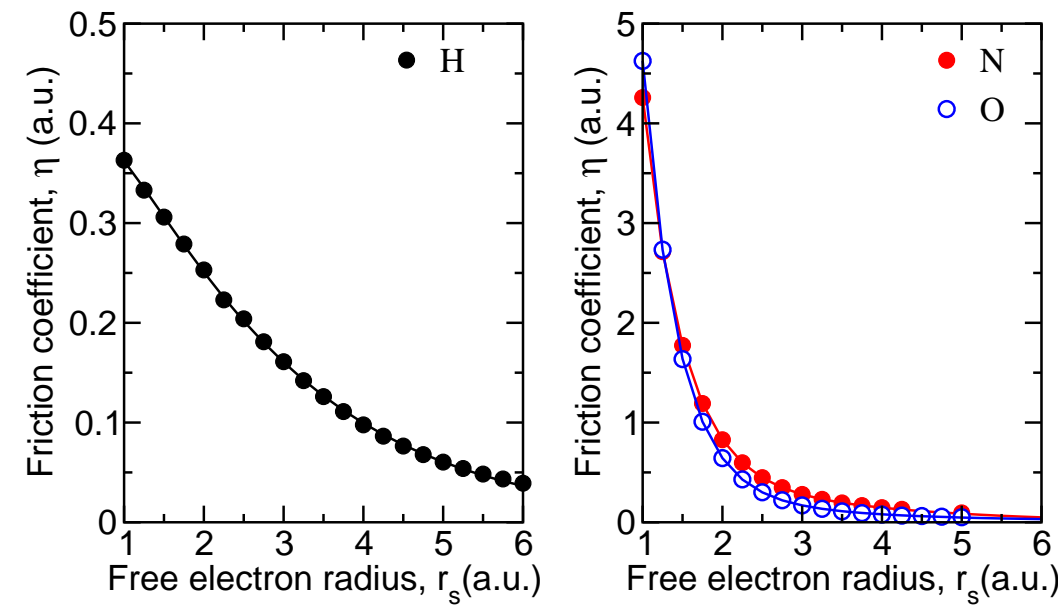

Fig. 1 Friction coefficients for an atom $(\mathrm{H}, \mathrm{N}, \mathrm{O})$ embedded in a free electron gas with density $\frac{1}{n_{0}}=\frac{4}{3} \pi r_{s}^{3}$. The values obtained from DFT using Eqs. (1) and (2) (symbols) are compared with the analytical fitting function of Eq. (3) (solid lines).

In the particular case of a diatomic molecule and assuming the frozen surface approximation ${ }^{1}$, the classical equations of motion for each atom of the impinging gas-molecule read within the LDFA model $[14,30]$

$$
\frac{d^{2} \mathbf{r}_{\mathbf{i}}}{d t^{2}}=-\frac{1}{m_{i}} \nabla_{i} V\left(\mathbf{r}_{i}, \mathbf{r}_{j}\right)-\eta\left(\mathbf{r}_{i}\right) \frac{d \mathbf{r}_{i}}{d t} \quad \text { with } i, j=1,2 \text { and } i \neq j
$$

where the first term in the right-hand side is the adiabatic force obtained from the six-dimensional (6D) PES $V\left(\mathbf{r}_{i}, \mathbf{r}_{j}\right)$ and the second term is the dissipative force experienced by each atom in the molecule. In this expression, $\mathbf{r}_{i, j}$ are the vector positions of the gas-atoms $i$ and $j$ conforming the molecule and $m_{i}$ is the mass of the atom $i$. As explained above, the friction coefficient depends at each point of the trajectory $r_{i}$ on the value that the electronic density of the bare metal surface would

\footnotetext{
${ }^{1}$ Within the frozen surface approximation, the multidimensional gas-surface PES is calculated fixing the positions of the surface atoms to the relaxed structure of the pristine surface. The PES dimensionality is then $3 n$, being $n$ the number of atoms in the impinging gas-molecule
} 
Table 1 Values of the parameters used in Eq. (3) that fit the dependence of the friction coefficients on the electronic density.

\begin{tabular}{lccccc}
\hline Atom & $\mathrm{n}$ & $\mathrm{i}$ & $A_{i}$ & $B_{i}$ & $C_{i}$ \\
\hline $\mathrm{H}$ & 1 & 1 & 0.633 & 0.275 & 0.558 \\
\hline $\mathrm{N}$ & 2 & 1 & 39.298 & -0.127 & 0.838 \\
& & 2 & -34.62 & 0.333 & 0.999 \\
\hline \multirow{2}{*}{$\mathrm{O}$} & 2 & 1 & 1.365 & -1.8287 & 0.082 \\
& & 2 & 80.342 & 0.4913 & 2.704 \\
\hline
\end{tabular}

take at this position. The case of a single atom scattering is obtained in a straightforward way by using the corresponding 3D atomic PES $V\left(\mathbf{r}_{i}\right)$. Finally, note that the adiabatic dynamics calculation corresponds to neglecting the dissipative force in these equations.

\subsection{Generalized Langevin Oscillator model}

The GLO model was originally developed as an efficient theoretical framework for dealing with energy exchange and dissipation between the impinging atom/molecule and the lattice in elementary gas-surface dynamical processes [17, 18]. Combining gas-phase classical trajectory methods with statistical mechanics, the model permits to reduce the many-body equations of motion associated with the solid and focus on the local region of the surface that will actually interact with the gas-atom/molecule. The remaining lattice atoms take part by providing a harmonic heat bath. The reader is referred to the seminal works by Adelman and Doll [17] and Tully [18] for a full description and derivation of the model. In this subsection, the model is briefly overviewed following the implementation of Ref. [27] that uses for treating the gassurface interaction a multidimensional PES calculated within the frozen surface approximation.

Let us consider the particular case of a diatomic molecule colliding with a surface. In the GLO model, the dynamics of the whole system is reduced to a four-body problem that consist of the two atoms in the molecule, one three-dimensional (3D) harmonic oscillator representing the surface, and a second 3D harmonic oscillator (denoted as the ghost oscillator in the following) that provides the thermal bath. The former harmonic oscillator plays the role of the primary atoms in the original GLO model, while the ghost oscillator does it of the secondary atoms. The classical equations of motion for the transformed four-body system are the following, 


$$
\begin{aligned}
\frac{d^{2} \mathbf{r}_{i}}{d t^{2}} & =-\frac{1}{m_{i}} \nabla_{i} V\left(\mathbf{r}_{i}-\mathbf{r}_{s}, \mathbf{r}_{j}-\mathbf{r}_{s}\right) \quad \text { with } i, j=1,2 \text { and } i \neq j \\
\frac{d^{2} \mathbf{r}_{s}}{d t^{2}} & =-\frac{1}{m_{s}} \nabla_{s} V\left(\mathbf{r}_{i}-\mathbf{r}_{s}, \mathbf{r}_{j}-\mathbf{r}_{s}\right)-\hat{\omega}_{s}^{2} \mathbf{r}_{s}+\hat{\lambda}_{g s} \mathbf{r}_{g} \\
\frac{d^{2} \mathbf{r}_{g}}{d t^{2}} & =-\hat{\omega}_{g}^{2} \mathbf{r}_{g}+\hat{\lambda}_{g s} \mathbf{r}_{s}-\hat{\gamma}_{g} \frac{d \mathbf{r}_{g}}{d t}+\frac{1}{m_{s}} \mathbf{F}_{r}(t) .
\end{aligned}
$$

The first equation represents the motion of the gas-molecule due to the adiabatic force obtained from the ab-initio 6D PES, $V\left(\mathbf{r}_{i}-\mathbf{r}_{s}, \mathbf{r}_{j}-\mathbf{r}_{s}\right)$. The position of the surface denoted by $\mathbf{r}_{s}$ represents the rigid displacement of the surface respect to the equilibrium position as dictated by the $3 \mathrm{D}$ surface harmonic oscillator. Hence, this equation assumes that the adiabatic force depends instantaneously on the position of the gas-molecule respect to the moving surface. The second and third equations represent the surface motion in terms of a $3 \mathrm{D}$ harmonic oscillator with coordinates $\mathbf{r}_{s}$ and associated $3 \times 3$ frequency matrix $\hat{\omega}_{s}$ and the $3 \mathrm{D}$ ghost oscillator with coordinates $\mathbf{r}_{g}$ and associated frequency matrix $\hat{\omega}_{g}$. In both cases, the mass is taken equal to the mass of one surface atom, $m_{s}$. Energy exchange of the surface with the bulk is included through the coupling matrix $\hat{\lambda}_{g s}$ between the surface and ghost oscillators. Equation 7 shows that the ghost oscillator is also subject to a friction force with damping matrix $\hat{\gamma}_{g}$ and to a random force $\mathbf{F}_{r}(t)$ related to each other through the second fluctuation-dissipation theorem. In particular (see Refs. $[17,18]), \mathbf{F}_{r}(t)$ is a white noise Gaussian random force with variance $\left(2 k_{B} T_{s} \gamma_{g} / m_{s} \Delta t\right)^{1 / 2}$, where $k_{B}$ is the Boltzmann constant, $T_{S}$ is the surface temperature, and $\Delta t$ the integration time step used in the simulations. All these expressions show that the ghost oscillator is acting as a thermal bath that keeps the surface at the temperature $T_{s}$ : the friction force represents the energy dissipated from the surface to the bulk, while the random force assures the energy flow from the bulk to the surface due to the thermal vibrations of the lattice.

Following Ref. [27], the frequency matrices $\hat{\omega}_{s}$ and $\hat{\omega}_{g}$ associated to the surface and ghost oscillators are taken equal and diagonal. Under such condition, the elements of the frequency matrices and the coupling matrix are reduced to

$$
\left(\hat{\omega}_{s}^{2}\right)_{\alpha \alpha}=\left(\hat{\omega}_{g}^{2}\right)_{\alpha \alpha}=2 \omega_{\alpha}^{2} \quad \text { and } \quad\left(\hat{\lambda}_{g s}\right)_{\alpha \alpha}=\omega_{\alpha}^{2}
$$

where $\omega_{\alpha}$ denotes the oscillator frequencies for motion along the direction $\alpha=$ $x, y, z$. The values can easily be obtained from experimental o computed data as the surface phonon frequencies close to the Brillouin zone edges of the metal surface. The diagonal damping matrix is calculated from the Debye frequency $\omega_{D}$ as proposed in Ref. [18]:

$$
\hat{\gamma}_{g}=\frac{\pi}{6} \omega_{D} \mathbf{I}
$$

with I being the unit matrix. It is important to remark that for the common gassurface problems of interest, the GLO results do not require an accurate selection of the input parameters. 


\subsection{Combining the generalized Langevin oscillator and the local density friction models}

In the combined GLO\&LDFA model, the classical equations of motion for each atom of the molecule interacting with a surface given by Eq. (5) in section 2.2 are replaced by:

$$
\frac{d^{2} \mathbf{r}_{i}}{d t^{2}}=-\frac{1}{m_{i}} \nabla_{i} V\left(\mathbf{r}_{i}-\mathbf{r}_{s}, \mathbf{r}_{j}-\mathbf{r}_{s}\right)-\frac{1}{m_{i}} \eta\left(\mathbf{r}_{i}-\mathbf{r}_{s}\right) \frac{d \mathbf{r}_{i}}{d t}
$$

The first term on the right hand side is the adiabatic force acting on each gas-atom $i$, as before. The new second term on the right hand side consists of the dissipative force that accounts for electron-hole pair excitations within the LDFA explained in section 2.1. As explained there, the friction coefficient $\eta$ is that of the same atom $i$ moving in an homogeneous free electron gas with electronic density equal to that of the surface at the position at which the atom is placed. Since the surface is moving as a 3D harmonic oscillator, the actual position of the gas atom $i$ respect to the surface is $\mathbf{r}_{i}-\mathbf{r}_{s}$. This correction is included when evaluating the adiabatic force and the friction coefficient $\eta$ in the GLO\&LDFA model and explains the differences with Eq. (4).

The surface motion is represented as done in the GLO model:

$$
\begin{aligned}
& \frac{d^{2} \mathbf{r}_{s}}{d t^{2}}=-\frac{1}{m_{s}} \nabla_{s} V\left(\mathbf{r}_{i}-\mathbf{r}_{s}, \mathbf{r}_{j}-\mathbf{r}_{s}\right)-\hat{\omega}_{s}^{2} \mathbf{r}_{s}+\hat{\lambda}_{g s} \mathbf{r}_{g} \\
& \frac{d^{2} \mathbf{r}_{g}}{d t^{2}}=-\hat{\omega}_{g}^{2} \mathbf{r}_{g}+\hat{\lambda}_{g s} \mathbf{r}_{s}-\hat{\gamma}_{g} \frac{d \mathbf{r}_{g}}{d t}+\frac{1}{m_{s}} \mathbf{F}_{r}(t) .
\end{aligned}
$$

All together, the scheme set by Eqs. (8), (9) and (10) allows to incorporate electronic excitations induced by the gas-molecule via a friction force and phonon excitations/deexcitations via the coupling to the surface oscillator which is itself coupled to a thermal bath at $T_{s}$. Note that if the electronic friction term in Eq. (8) is neglected, the original GLO model is recovered. The LDFA equations of motion can be obtained by setting $\mathbf{r}_{s}=0$ in Eq. (8). Obviously, the adiabatic calculation corresponds to solving Eq. (8) with $\eta\left(\mathbf{r}_{i}\right)=0$ and $\mathbf{r}_{s}=0$.

\section{Results on some representative systems}

The theoretical models described in previous section have been applied to explore the contribution of electronic and/or phonon excitations in the relevant gas-surface elementary processes: dissociation, scattering, and atomic and molecular adsorption. The main conclusions derived from those studies are briefly reviewed in this section. 
Dissociative adsorption

The LDFA model has been used to investigate the role of the $e$ - $h$ pair excitations in the dissociation of $\mathrm{H}_{2}$ on $\mathrm{Cu}(110)$. Experimentally, the dissociative adsorption of $\mathrm{H}_{2} / \mathrm{Cu}(110)$ is activated $[38,39]$, i.e. there is a minimum incidence energy of the $\mathrm{H}_{2}$ molecules to observe dissociation. Above this energy, the dissociation probability increases with energy following a characteristic 'S-like' curve. Classical dynamics calculations performed on an accurate 6D DFT-based PES showed that the $\mathrm{H}_{2}$ dissociation is ruled by a late activation barrier at short distances from the surface [4]. It is at these distances where the molecule finds high electron densities and where larger energy losses are more likely to occur. Inclusion of the electron-hole pair excitations showed, however, that this mechanism only implies a slight reduction of the dissociative sticking probabilities, as shown in Fig. 2. The reduction is obtained because the inclusion of a pure energy loss channel prevents some of the molecules to overcome the late activation barriers.

Fig. 2 Dissociative sticking probability of $\mathrm{H}_{2}$ on $\mathrm{Cu}(110)$ as obtained from the adiabatic approximation (open circles) and from the LDFA model (filled red circles), for different incidence angles [14]. Simulations performed within the frozen surface approximation using a 6D DFT-based PES. Lines shown to guide the eye.

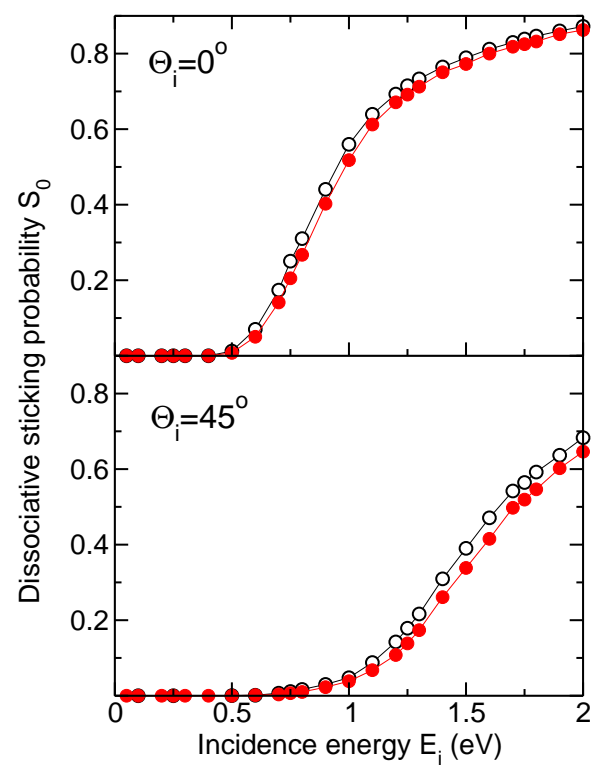

The dissociative dynamics of $\mathrm{N}_{2}$ on the two $\mathrm{W}$ surfaces is more involved. Molecular beam experiments showed that $\mathrm{N}_{2} / \mathrm{W}(110)$ [40] is activated, while the initial sticking coefficient of $\mathrm{N}_{2} / \mathrm{W}(100)$ measured at different surface temperatures exhibits the typical features associated to non-activated dissociation [41]. These observations have been reasonably reproduced by $6 \mathrm{D}$ dynamics simulations performed on accurate PESs calculated with DFT. On both surfaces, the dissociation process combines direct and trapping mediated mechanisms depending on the $\mathrm{N}_{2}$ incidence conditions [1]. The lower reactivity on $\mathrm{W}(110)$ is caused by the reduced configurational space leading to dissociation as compare with that on $\mathrm{W}(100)$. The existence 
of the trapping mechanism, in which the molecules stay bouncing off several times close to the surface before dissociating or being reflected, provides the ideal conditions for causing innumerable electronic excitations. Under these circumstances, the dissociation process is expected to increase. Adding a dissipation channel makes more difficult to recover the perpendicular translational energy necessary to escape from the surface. As observed in Fig. 3, the effect of including electron-hole pair excitations is to increase the trapping mechanism, while keeping the direct channel almost unchanged. However, the increase observed in the total dissociation probability, which practically coincides with that obtained through the trapping-mediated channel, is rather minor. Compared with the adiabatic calculation $S_{0}$ increases in only $1-5 \%$ as a result of including electronic excitations.

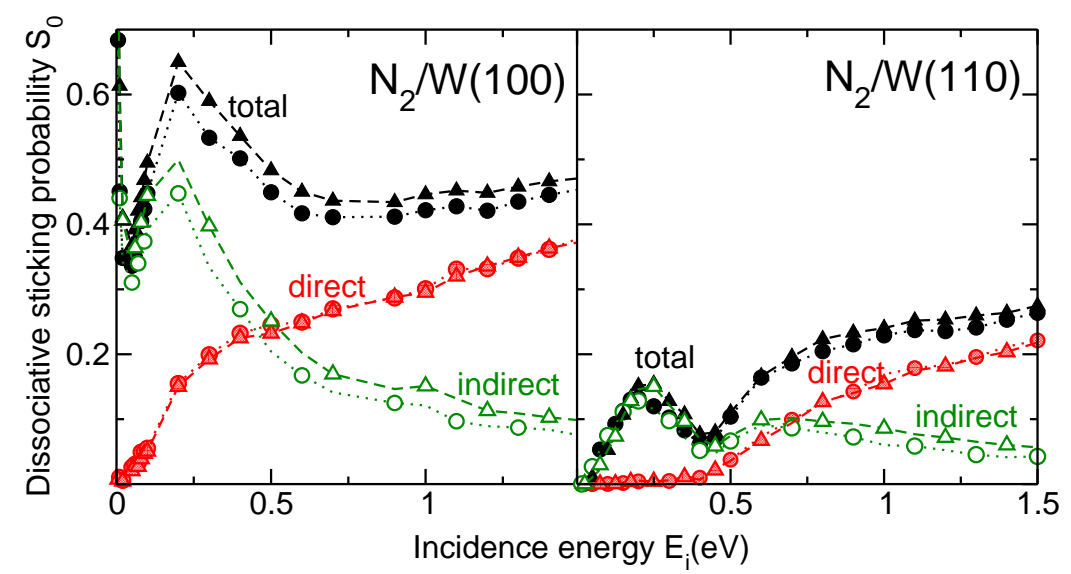

Fig. 3 Dissociative sticking probability of $\mathrm{N}_{2}$ on W(100) (left panel) and on W(110) (right panel) as obtained with (triangles) and without (circles) electronic friction in the $6 \mathrm{D}$ classical dynamics simulations. Contributions to $S_{0}$ coming from the direct and the trapping-mediated channels are represented by shadowed and open symbols, respectively. Lines shown to guide the eye.

These calculations show that the effect of electronic friction in the dissociative dynamics is in all cases very minor, despite the friction coefficients close to the surface can be large. The reason is that the dissipative force is not only proportional to the friction coefficient, but also to the projectile velocity. In the region where the electron density is high, the molecule-surface potential is highly repulsive, what produces a substantial reduction of the molecular kinetic energy. As a result, the dissipative force is small and so the energy loss that seems insufficient to affect the dynamics in a significant way.

\section{Molecular and atomic scattering}

The analysis of the reflected molecules performed on [30] with the LDFA model corroborates the marginal energy losses caused by $e-h$ pair excitations and provides 
abundant quantitative information on this respect. As an example, Fig. 4 shows the energy loss distributions for $\mathrm{N}_{2}$ scattered off the $\mathrm{W}(100)$ and $\mathrm{W}(110)$ surfaces. The observed two peak structure can be related to the distances of closest approach to the surface $Z_{\text {min }}$ probed by the reflected molecules. As a general trend, molecules contributing to the high-energy loss peak probe distances close to the surface, where the electronic density is high. In contrast, the low-energy peaks corresponds to molecules that are reflected at larger distances from the surface, i.e. in regions of low electron density. This can be observed in the insets of Fig. 4, which show the $Z_{\text {min }}$ distributions for the trajectories that give rise to the low-energy loss peaks (shadow bars) and for the ones that give rise to the high-energy loss peaks (open bars). The study performed in [30] also shows that there is a correlation between the number of rebounds and the energy loss, since a larger number of rebounds implies longer interaction times and hence larger energy losses. This correlation explains why the high-energy loss peak of the W(100) energy loss spectra appears at larger energy losses than on the W(110).

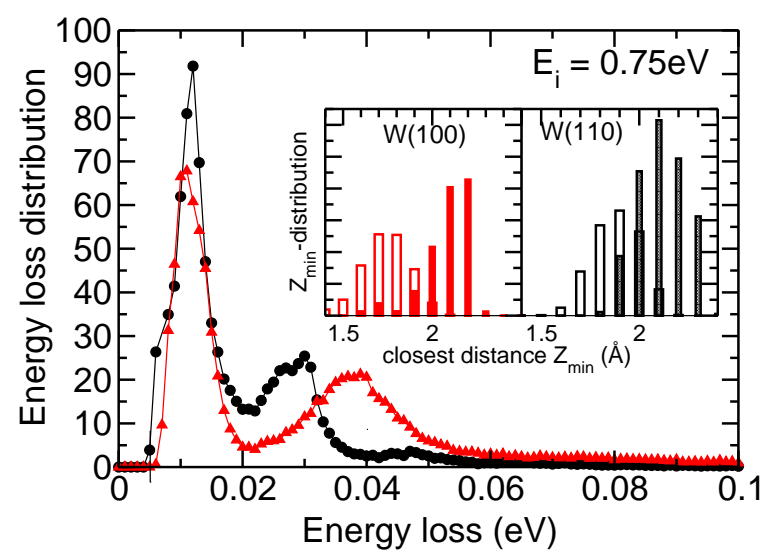

Fig. 4 Energy loss spectra of the $\mathrm{N}_{2}$ molecules reflected from W(110) (circles) and W(100) (triangles). For each surface, the insets show the closest approach distance distribution of those reflecting molecules that contribute to the low-energy (shadowed bars) and high-energy (open bars) loss peaks. Results for normal incidence and $\mathrm{E}_{i}=0.75 \mathrm{eV}$. Lines shown to guide the eye.

Still the energy dissipation due to electronic friction is insufficient to explain the energy losses reported in [42]. Hanisco et al. find that when a rotationally cold $\mathrm{N}_{2}$ beam is scattered off $\mathrm{W}(110)$, the molecules remaining in a low rotational state upon reflection lose around $30 \%$ of their incidence energy. As the exit rotational state increases, the energy lost of these molecules decreases. Classical molecular dynamics simulations performed in [28] show that the observed energy loses are basically due to energy exchange with the lattice. This is illustrated in Fig. 5 where we compare the experiments by Hanisco et al. with the results of the different simulations. The figure shows the average exit translational energy as a function of the exit rotational energy for the scattered molecules, at normal incidence and detection angles. The 
decrease of the final average translational energy with increasing rotational energy obtained with the adiabatic calculation reflects how the rotational excitation occurs at expense of translational energy as a consequence of the PES corrugation. Inclusion of $e-h$ pair excitations produces a marginal energy loss that, in contrast to the experimental data, does not depend on the rotational energy. Indeed it is necessary to include energy exchange with the lattice in order to reproduce the experimental observation that more energy is lost at low exit rotational states. The similar results obtained with the GLO and the GLO\&LDFA models remark the predominant role of phonon excitations in these kind of experiments.

Fig. 5 Exit translational energy of $\mathrm{N}_{2}$ scattered off $\mathrm{W}(110)$ as a function of the exit rotational energy, for normal incidence and detection angles and two incidence energies $E_{i}$. In triangles, the experimental data of [42]. Results obtained from various $6 \mathrm{D}$ classical dynamics simulations: adiabatic calculation (open circles), LDFA (filled circles), GLO (open squares), and GLO\&LDFA (filled squares). Lines shown to guide the eye. The surface temperature is $T_{s}=1200 \mathrm{~K}$.
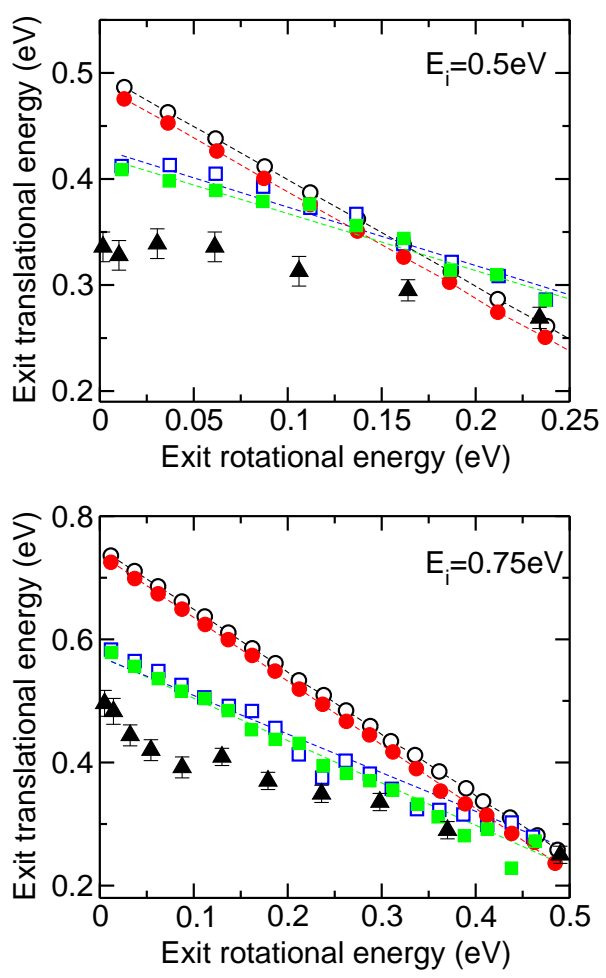

Phonon excitations as compared with electron-hole pair excitations are also dominant in the scattering of hyperthermal $\mathrm{N}$ atoms on $\mathrm{Ag}(111)$. Experiments performed using an effusive beam with an average energy of $4.3 \mathrm{eV}$ and a FWHM of $\sim 5.0 \mathrm{eV}$ measured different energy losses depending on the scattering angle of the reflected $\mathrm{N}$ atoms [43]. The data showing the ratio between the average final $\left\langle E_{f}\right\rangle$ and initial $\left\langle E_{i}\right\rangle$ energies as a function of the in-plane scattering angle $\Theta_{t}$ is depicted in Fig. 6, together with the experimental setup and the effusive beam profile. The experiments show a decrease of the final average energy as the scattering angle increases. A remarkable feature is the sharp increase of the energy ratio at $\Theta_{t}<60^{\circ}$, i.e., for grazing outgoing angles. At this point, the use of an adequate description 
of the experimental effusive beam in the simulations is crucial to understand such dependence. In particular, the energy ratios larger than one measured for small $\Theta_{t}$ are a consequence of the decrease in the number of the low energy atoms for that outgoing angles [28]. However, Fig. 6 shows that quantitative agreement with the experiments is obtained only with the GLO and GLO\&LDFA simulations, i.e., when energy exchange with the lattice is allowed.
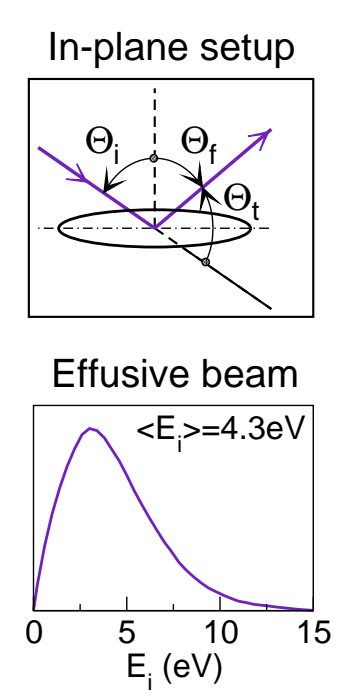

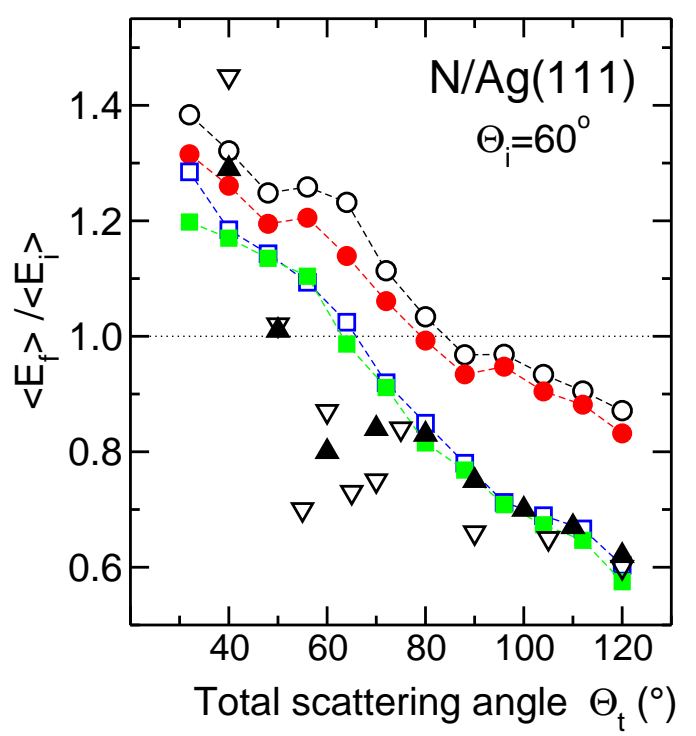

Fig. 6 Angle-resolved ratios of final to initial energy $\left.\left(<E_{\mathrm{f}}>/<E_{\mathrm{i}}\right\rangle\right)$ plotted as a function of the total in-plane scattering angle $\left[\Theta_{t}=180^{\circ}-\left(\Theta_{i}+\Theta_{f}\right)\right.$ as depicted in the upper-left panel] for $\mathrm{N}$ atoms with $\Theta_{i}=60^{\circ}$ scattered from $\operatorname{Ag}(111)$ at $T_{s}=500 \mathrm{~K}$. The experimental data of [43] (open and filled triangles) are compared with the results of various simulations [28]: adiabatic calculation (open circles), LDFA (filled circles), GLO (open squares), and GLO\&LDFA (filled squares). The bottom-left panel shows the shape of the experimental and theoretical effusive beam.

\section{Molecular and atomic adsorption}

Inclusion of energy dissipation mechanisms are mandatory in the study of atomic and molecular adsorption on surfaces. During such process, the atom/molecule loses energy up to be accommodated in the adsorption well. Therefore, one should go beyond the adiabatic and frozen surface approximations in order to describe them. Energy exchange with the lattice through the GLO model was first implemented in a 6D DFT-based PES by Busnengo et al. [26,27] to study the adsorption of $\mathrm{H}_{2}$ on $\operatorname{Pd}(110)$. The authors find that the appearance of such process in the simulations is a direct consequence of the dissipation mechanism added in the GLO through the random and friction forces. Compared with the more simple surface oscillator 
model that allows energy exchange to and from the surface, but conserving the total energy of the system, the GLO is a clear improvement that provides a more realistic description of surface temperature effects in gas-surface dynamics.

The GLO model has been also used to study the adsorption of $\mathrm{N}_{2}$ on $\mathrm{Fe}(110)$, which is characterized by the existence of two adsorption states [44] and on $\mathrm{W}(110)$, for which a unique molecular adsorption state is identified. Figure 7 shows the adsorption probability as a function of $E_{i}$ for $T_{s}$ varying in the range $80-500 \mathrm{~K}$. On both surfaces, $\mathrm{N}_{2}$ adsorption tends to decrease as $T_{s}$ increases. Since the molecules are hot compared to the surface (i.e. $E_{i}>k_{B} T_{s}$ ), energy is transferred in average from the former to the latter what favors the adsorption process. As $T_{s}$ increases, the surface-molecule energy difference becomes smaller and less energy is dissipated from the molecule to the lattice. Thus, the possibility of trapping on the adsorption well is reduced. Focusing on the $E_{i}$-dependence, we observe that the curves exhibit a similar shape. The adsorption probabilities increase monotonously with $E_{i}$ up to reaching a maximum and then decrease when the energy exchange with the lattice is insufficient for the molecules to lose all the energy required to accommodate in the adsorption wells. The appearance of a threshold energy and the increase obtained at low energies is due to the presence of energy barriers in the entrance channel that the molecules have to overcome to arrive at the adsorption wells.

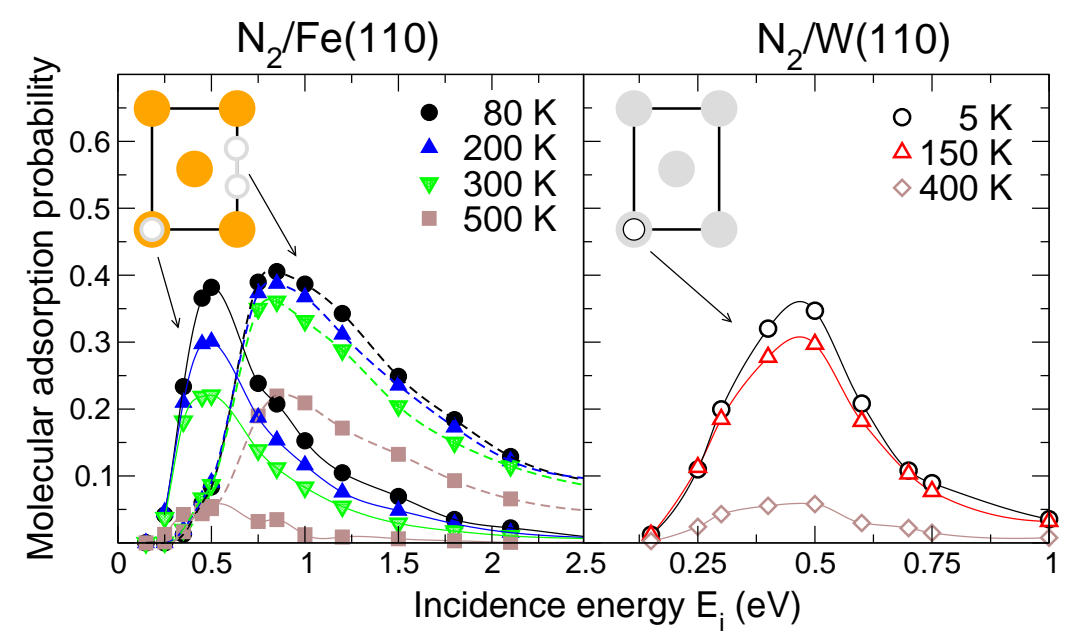

Fig. 7 Molecular adsorption probability as a function of the incident energy, for different surface temperatures and normal incidence: (a) $\mathrm{N}_{2}$ adsorbed on $\mathrm{Fe}(110)$, solid and dashed lines correspond to adsorption on the top-vertical and hollow-parallel wells found on this surface and (b) $\mathrm{N}_{2}$ adsorbed on the top-vertical well of W(110).

A detailed analysis on the role of electron and phonon excitations in the adsorption of $\mathrm{N}$ on $\operatorname{Ag}(111)$ is reported in [45]. According to the various simulations performed in this system (LDFA, GLO and GLO\&LDFA), phonons are responsible for determining the adsorption probability but electronic excitations are relevant at a 
later stage to finally accommodate the $\mathrm{N}$ atoms on the adsorption sites. These results remark the importance of using a theoretical model that intrinsically combines both energy dissipation channels to properly describe the full dynamics of the adsorption process.

\section{Conclusions}

The theoretical framework presented in this chapter allows to include both phonon and electron-hole pair excitations in simulations of gas-surface collisions, keeping the accuracy of an ab-initio PES to treat the particle/surface interaction. The method is easy to implement and flexible enough to disentangle the relevance of nonadiabatic effects in a variety of situations.

From the results that, using this method, we have obtained for different systems some general conclusions can be extracted:

- In order to model realistically the gas-surface dynamics it is fundamental to take into account the full-dimensionality of the problem and to describe the interaction with an ab-initio PES. In fact, in some cases, an adiabatic calculation, that excludes electronic excitations and energy exchange with the lattice, reproduces satisfactorily the experimental results. In these cases, the inclusion of inelastic channels represents just a minor effect.

- In general, energy exchange with the lattice and phonon excitations are more important than electron-hole pair excitations in the range of energies of interest. In fact, reaction probabilities and properties of scattered particles are marginally affected by the inclusion of the electronic excitation channel.

- Therefore, the measured energy loss of scattered thermal and hyperthermal molecules is the result of the energy that they exchange with the lattice. Nevertheless, even in this case, a proper description of the energy and angular distributions of the scattered species requires to take into account the full dimensionality of the problem and a realistic PES.

- Finally, for the description of molecular and atomic adsorption processes that require the accommodation of the projectile in the corresponding adsorption well the inclusion of inelastic effects is mandatory. In this case, at short times, the energy loss is dominated by phonon excitations, and the efficiency of this mechanism determines whether the particle is adsorbed or not. Nevertheless, interestingly, it is the electron-hole pair excitation mechanism the one that governs the accommodation of the particle in the adsorption well at longer times

Acknowledgements This work has been supported in part by the Basque Departamento de Educación, Universidades e Investigación, the University of the Basque Country UPV/EHU (Grant No. IT-366-07) and the Spanish Ministerio de Ciencia e Innovación (Grant No. FIS2010-19609$\mathrm{C} 02-02)$. 


\section{References}

1. M. Alducin, R. Díez Muiño, H. F. Busnengo, and A. Salin, Phys. Rev. Lett. 97, 056102 (2006).

2. C. Díaz, J. K. Vincent, G. P. Krishnamohan, R. A. Olsen, G. J. Kroes, K. Honkala, and J. K. Nørskov, Phys. Rev. Lett. 96, 096102 (2006).

3. P. Nieto, E. Pijper, D. Barredo, G. Laurent, R. A. Olsen, E.-J. Baerends, G.-J. Kroes, and D. Farías, Science 312, 86-89 (2006).

4. A. Salin, J. Chem. Phys. 124, 104704 (2006).

5. C. Díaz, E. Pijper, R. A. Olsen, H. F. Busnengo, D. J. Auerbach, and G. J. Kroes, Science 326, 832 (2009).

6. K. R Geethalakshmi, J. I Juaristi, R. Díez Muiño, and M. Alducin, Phys. Chem. Chem. Phys. 13, 4357 (2011).

7. I. Goikoetxea, J. Beltrán, J. Meyer, J I Juaristi, M. Alducin, and K. Reuter, New J. Phys. 14, 013050 (2012).

8. B. Gergen, H. Nienhaus, W. H. Weinberg, and E. W. McFarland, Science 294, 2521 (2001).

9. J. D. White, J. Chen, D. Matsiev, D. J. Auerbach, and A. M. Wodtke, Nature 433, 503 (2005).

10. D. Krix, R. Nünthel, and H. Nienhaus, Phys. Rev. B 75, 073410 (2007).

11. I. Rahinov, R. Cooper, D. Matsiev, C. Bartels, D. J. Auerbach, and A. M. Wodtke, Phys. Chem. Chem. Phys. 13, 12680 (2011).

12. M. Head-Gordon and J. C. Tully, J. Chem. Phys. 103, 10137 (1995).

13. J. R. Trail, D. M. Bird, M. Persson, and S. Holloway, J. Chem. Phys. 119, 4539 (2003).

14. J. I. Juaristi, M. Alducin, R. Díez Muiño, H. F. Busnengo, and A. Salin, Phys. Rev. Lett. 100, $116102(2008)$.

15. N. Shenvi, S. Roy, and J. C. Tully, Science 326, 829 (2009).

16. S. Monturet and P. Saalfrank, Phys. Rev. B 82, 075404 (2010).

17. S. A. Adelman and J. D. Doll, J. Chem. Phys. 64, 2375 (1976).

18. J. C. Tully, J. Chem. Phys. 73, 1975 (1980).

19. J. R. Manson, Phys. Rev. B 43, 6924 (1991).

20. M. Dohle and P. Saalfrank, Surf. Sci. 373, 95 (1997).

21. J. R. Manson, Phys. Rev. B 58, 2253 (1998).

22. A. Muis and J. R. Manson, J. Chem. Phys. 111, 730 (1999).

23. W. W. Hayes and J. R. Manson, Phys. Rev. B 75, 113408 (2007).

24. H. Ambaye, J. R. Manson, O. Weisse, C. Wesenberg, M. Binetti, and E. Hasselbrink, J. Chem. Phys. 121, 1901 (2004).

25. H. Ambaye and J. R. Manson, J. Chem. Phys. 125, 084717 (2006).

26. H. F. Busnengo, W. Dong, and A. Salin, Phys. Rev. Lett. 93, 236103 (2004).

27. H. F. Busnengo, M. A. Di Césare, W. Dong, and A. Salin, Phys. Rev. B 72, 125411 (2005).

28. L. Martin-Gondre, M. Alducin, G. A. Bocan, R. Díez Muiño, and J. I. Juaristi, Phys. Rev. Lett. 108, 096101 (2012).

29. C. O. Almbladh, U. von Barth, Z. D. Popovic, and M. J. Stott, Phys. Rev. B 14, 2250 (1976).

30. I Goikoetxea, J I Juaristi, M Alducin, and R. Díez Muiño, J. of Phys.: Cond. Matt. 21, 264007 (2009).

31. A. Salin, A. Arnau, P. M. Echenique, and E. Zaremba, Phys. Rev. B 59, 2537 (1999).

32. K. Schönhammer, Phys. Rev. B 37, 7735 (1988).

33. L. Bönig and K. Schönhammer, Phys. Rev. B 39, 7413 (1989).

34. P.M. Echenique, R.M. Nieminen, and R.H. Ritchie, Solid State Communications 37, 779 (1981).

35. P. M. Echenique, R. M. Nieminen, J. C. Ashley, and R. H. Ritchie, Phys. Rev. A 33, 897 (1986).

36. J. I. Juaristi, A. Arnau, P. M. Echenique, C. Auth, and H. Winter, Phys. Rev. Lett. 82, 1048 (1999).

37. E Zaremba, J H Rose, L M Sander, and H B Shore, J. of Phys. F: Metal Physics 7, 1763 (1977).

38. G. Anger, A. Winkler, and K.D. Rendulic, Surface Science 220, 1 (1989).

39. H.F. Berger and K.D. Rendulic, Surface Science 253, 325 (1991). 
40. H. E. Pfnur, C. T. Rettner, J. Lee, R. J. Madix, and D. J. Auerbach, J. Chem. Phys. 85, 7452 (1986).

41. C. T. Rettner, H. Stein, and E. K. Schweizer, J. Chem. Phys. 89, 3337 (1988).

42. T. F. Hanisco and A. C. Kummel, J. Vac. Sci. Technol. A 11, 1907 (1993).

43. H. Ueta, M. A. Gleeson, and A. W. Kleyn, J. Phys. Chem. A 113, 15092 (2009).

44. I. Goikoetxea, M. Alducin, R. Díez Muiño, and J. I. Juaristi, Phys. Chem. Chem. Phys. 14, 7471 (2012).

45. L. Martin-Gondre, G. A. Bocan, M. Alducin, J. I Juaristi, and R. Díez Muiño, Computational and Theoretical Chemistry 990, 126 (2012). 